\section{Membership Query}

Mirella M. Moro

Departamento de Ciencia da Computaçao, Universidade Federal de Minas Gerais - UFMG, Belo Horizonte, MG, Brazil

\section{Synonyms}

Equality query; Equality selection

\section{Definition}

Consider a relation $R$ whose schema contains some attribute $A$ taking values over a domain $D$. A membership query retrieves all tuples in $R$ with $A=x(x \in D)$.

\section{Key Points}

A membership query effectively checks membership in a set (relation). As such, it can be implemented using either a hash-based index (built on the attribute(s) involved in the query) or a B+-tree. If a hashing scheme is used, each indexed value is placed on an appropriate hash bucket. Then all records that satisfy $A=x$ are located on the bucket responsible for value $x$. If $A$ is a numeric attribute (and can thus be indexed using an order-preserving access method like a $\mathrm{B}+-$ tree), the membership query is a special case of a range query where the range interval [low, high] is reduced to a single value $($ low $=h i g h=x)$.

\section{Cross-References}

- Access Methods

$>\mathrm{B}+$-Tree

- Hashing 